\title{
POISSON-GAUSSIAN NOISE PARAMETER ESTIMATION IN FLUORESCENCE MICROSCOPY IMAGING
}

\author{
Anna Jezierska ${ }^{1}$, Hugues Talbot ${ }^{1}$, Caroline Chaux ${ }^{1}$, Jean-Christophe Pesquet ${ }^{1}$, and Gilbert Engler ${ }^{2}$ \\ ${ }^{1}$ Université Paris-Est, LIGM, UMR CNRS 8049 - 77454 Marne-la-Vallée, France \\ \{first.last\}@univ-paris-est.fr \\ ${ }^{2}$ IBSV Unit, INRA - 06903 Sophia Antipolis, France \\ Gilbert.Engleresophia.inra.fr
}

\begin{abstract}
In this paper, we present a new fully automatic approach for noise parameter estimation in the context of fluorescence imaging systems. In particular, we address the problem of Poisson-Gaussian noise modeling in the nonstationary case. In microscopy practice, the nonstationarity is due to the photobleaching effect. The proposed method consists of an adequate moment based initialization followed by Expectation-Maximization iterations. This approach is shown to provide reliable estimates of the mean and the variance of the Gaussian noise and of the scale parameter of Poisson noise, as well as of the photobleaching rates. The algorithm performance is demonstrated on both synthetic and real macro confocal laser scanning microscope image sequences.
\end{abstract}

Index Terms - Noise identification, confocal imaging systems calibration, fluorescence photobleaching, Expectation-Maximization algorithm, image calibration

\section{INTRODUCTION}

The purpose of this work is to provide an estimation method for the noise parameters arising in fluorescence imaging systems. In many image restoration methods, these parameters are required to be known $[1,2]$. Moreover, noise parameters provide feedback concerning imaging conditions, and thereby they can be used in the microscope calibration process [3]. The noise sources were described from a physical viewpoint in [4], where also the corresponding statistical characteristics were studied. In practice, one usually considers simplified models e.g. either Gaussian [5] or Poisson [6].

Recently some works have begun investigating a more realistic Poisson-Gaussian model with non-zero mean. Previous noise parameter estimation studies in fluorescence imaging systems can be found in $[7,8,9,10,11,12]$. The author in [7] proposes a cumulantbased approach. The strategies proposed in $[9,10]$ rely on approximations of the Poisson-Gaussian noise statistics based on the Generalized Anscombe Transform. Then, the problem is addressed by using a simple regression based approach. In [11] authors show that the scale parameter of Poisson noise is not a pure estimate of the detector gain. In this work, a maximum likelihood method is proposed which sometimes leads to inaccurate results, due to an unreliable background classification scheme. Noise estimation from image sequences was previously considered in $[8,12]$. However, these methods were designed for specific fluorescence imaging systems, i.e. wide-field [8] and confocal microscopy [12].

This work was supported by the Agence Nationale de la Recherche under grant ANR-09-EMER-004-03.
In this paper, we propose a novel approach based on an Expectation-Maximization (EM) algorithm, following our previous work in [13]. We focus on fluorescence imaging systems, especially in the field of confocal microscopy and so-called macroscopy systems [14]. Similarly to [8, 12], as input data we use time-lapse fluorescence images, which consist of repeated images of motionless specimens at given times. The considered problem is more challenging than the one investigated in [13] due to the nonstationarity of the acquired signal caused by photobleaching. This effect is a process of intensity decay in time usually modeled with an exponentially decreasing function $[15,3]$. Although other photobleaching forms were also investigated [16], our analysis in this paper is based on the latter model, also known as three-parameter exponential model [17]. More specifically, in order to characterize the dependency between Poisson noise and photobleaching, we adopt the same model as the one presented in [6]. However, we relax the assumption of constant bleaching decay for the whole image, which is not realistic according to our experiments in the last section of this paper. Since the EM optimization framework is not guaranteed to converge to a global minimizer of the negative log-likelihood, its behavior can be improved by carefully setting its initialization. Usually the choice of a good starting value is discussed in the context of specific applications $[18,19,20,13]$. We address this key problem for Poisson-Gaussian parameter estimation in the presence of photobleaching by proposing an adequate moment based initialization. Moreover, we provide an extension of the EM approach developed in [13] in order to allow us to jointly estimate Poisson-Gaussian noise parameters, to reconstruct the original signal and to estimate its photobleaching rates.

The paper is organized as follows. We provide a description of the noise identification problem from a time-series standpoint in Section 2. We then present the proposed iterative method in Section 3. Section 4 illustrates the algorithm performance on synthetic data and real confocal macroscope image sequences obtained from biological samples. Section 5 concludes the paper.

\section{NOISE MODEL}

\subsection{Adopted scheme}

We consider data $\left(u_{s}\right)_{1 \leq s \leq S}$ where $s$ corresponds to a location index (e.g. locating pixel $(x, y)$ in $2 \mathrm{D}$ or voxel $(x, y, z)$ in $3 \mathrm{D})$, which are corrupted with a Poisson-Gaussian noise, and for which we observe $T$ realizations. Each realization will be indexed by the time index $t \in\{1, \ldots, T\}$. Such a framework leads us to the following model:

$$
(\forall s \in\{1, \ldots, S\})(\forall t \in\{1, \ldots, T\}) R_{s, t}=\alpha Q_{s, t}+W_{s, t}
$$


where $\alpha \in \mathbb{R}$ is a scaling parameter and $R_{s, t}$ is the observed random variable at time $t$ and location $s$. In the above model, the following auxiliary random variables intervenes

$$
\begin{aligned}
Q_{s, t} & \sim \mathcal{P}\left(u_{s} e^{-k_{s} t}\right) \\
W_{s, t} & \sim \mathcal{N}\left(c, \sigma^{2}\right)
\end{aligned}
$$

where $\left(u_{s}\right)_{1 \leq s \leq S} \in[0,+\infty)^{S}$ is the "clean" image (possibly resulting from a blur of the original image and some offset), $\left(k_{s}\right)_{1 \leq s \leq S} \in$ $(0,+\infty)^{S}$ denote the photobleaching rates, and $c \in \mathbb{R}($ resp. $\sigma>0)$ is the mean value (resp. standard-deviation) of the Gaussian noise.

\subsection{Considered problem}

The problem is to estimate $u=\left(u_{s}\right)_{1 \leq s \leq S}, k=\left(k_{s}\right)_{1 \leq s \leq S}, \alpha, c$ and $\sigma^{2}$ from the available observation vector $r=\left(r_{s, t}\right)_{1 \leq s \leq S, 1 \leq t \leq T}$, which is a realization of the random field $R=\left(R_{s, t}\right)_{1 \leq s \leq S, 1 \leq t \leq T}$. We have thus $2 S+3$ parameters to estimate. We define the vector of unknown parameters as $\theta=\left(u, k, \alpha, c, \sigma^{2}\right)$.

In the following, it is assumed that $u$ is deterministic and that $Q=\left(Q_{s, t}\right)_{1 \leq s \leq S, 1 \leq t \leq T}$ and $W=\left(W_{s, t}\right)_{1 \leq s \leq S, 1 \leq t \leq T}$ are mutually independent random fields. In addition, the components of $W$ (resp. $Q$ ) are assumed to be independent. These assumptions let us define the cumulant of order $n$ as $\kappa_{n}\left[R_{s, t}\right]=\alpha^{n} \kappa_{n}\left[Q_{s, t}\right]+$ $\kappa_{n}\left[W_{s, t}\right]$. This leads to the following results:

- mean value: $\quad \kappa_{1}\left[R_{s, t}\right]=\mathrm{E}\left[R_{s, t}\right]=\alpha e^{-k_{s} t} u_{s}+c$

- variance: $\quad \kappa_{2}\left[R_{s, t}\right]=\operatorname{Var}\left[R_{s, t}\right]=\alpha^{2} e^{-k_{s} t} u_{s}+\sigma^{2}$

- higher-order cumulants: $n \geq 3, \kappa_{n}\left[R_{s, t}\right]=\alpha^{n} u_{s} e^{-k_{s} t}$.

\section{NOISE ESTIMATION METHOD}

\subsection{Moment based method}

Several procedures may be derived from (4), (5), and (6) in order to estimate $\theta$, but they are not equally reliable. For example, according to our observations, $\frac{\kappa_{4}\left[R_{s, t}\right]}{\kappa_{3}\left[R_{s, t}\right]}$ does not provide a very accurate estimate of $\alpha$. Thus, we propose to define preliminary estimates of $\left(k_{s}\right)_{1 \leq s \leq S}, c$ and $\left(a_{s}=\alpha u_{s}\right)_{1 \leq s \leq S}$ by noticing that (4) can be reexpressed as

$$
R_{s, t}=a_{s} e^{-k_{s} t}+c+E_{s, t}
$$

where $\left(E_{s, t}\right)_{1 \leq s \leq S, 1 \leq t \leq T}$ are independent zero-mean random variables. This suggests to employ a nonlinear least squares approach to compute estimates $\widehat{a}=\left(\widehat{a}_{s}\right)_{1 \leq s \leq S}, \widehat{k}=\left(k_{s}\right)_{1 \leq s \leq S}, \widehat{c}$ of the parameters:

$$
(\widehat{a}, \widehat{k}, \widehat{c})=\underset{a, k, c}{\operatorname{argmin}} \sum_{s=1}^{S} \sum_{t=1}^{T} \omega_{s, t}\left(r_{s, t}-c-a_{s} e^{-k_{s} t}\right)^{2}
$$

where $\left(\omega_{s, t}\right)_{1 \leq s \leq S, 1 \leq t \leq T}$ are positive weights. In order to efficiently solve the associated minimization problem, we propose to use an alternating optimization method (see Algorithm 1). For every $s \in\{1, \ldots, S\}$, the minimization subproblem to be solved at each iteration reduces to a linear least squares one for a given value of $k_{s}$. The minimization procedure thus reduces to a one-variable search (over $k_{s}$ ) which can be performed by standard numerical methods, e.g. the Nelder-Mead simplex method [21].

The estimates provided by this method are sufficiently stable, as only first order statistics are used. These results allow us to provide

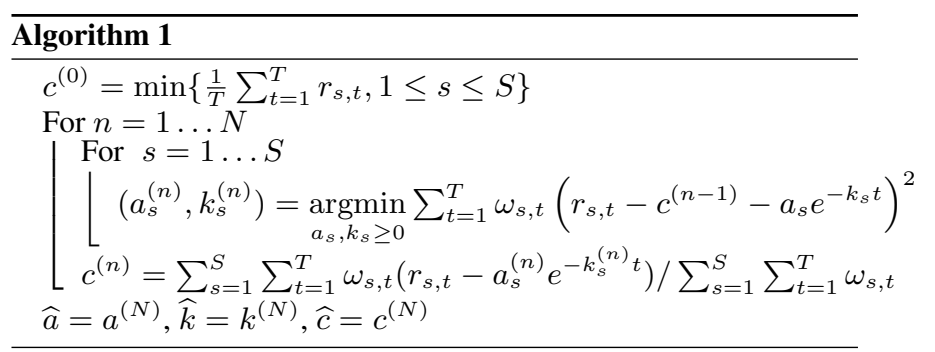

simple estimates of the remaining parameters. Indeed, by rewriting (5) as

$$
\mathrm{E}\left[\left(R_{s, t}-\mathrm{E}\left[R_{s, t}\right]\right)^{2}\right]=\alpha a_{s} e^{-k_{s} t}+\sigma^{2},
$$

the following least squares estimate for $\alpha$ can be derived:

$$
\widehat{\alpha}=\frac{\bar{\nu} \sum_{s=1}^{S} \nu_{s} m_{s} v_{s}-\sum_{s=1}^{S} \nu_{s} m_{s} \sum_{s=1}^{S} \nu_{s} v_{s}}{\bar{\nu} \sum_{s=1}^{S} \nu_{s} m_{s}^{2}-\left(\sum_{s=1}^{S} \nu_{s} m_{s}\right)^{2}},
$$

where $\left(\nu_{s}\right)_{1 \leq s \leq S}$ are positive weights, $\bar{\nu}=T \sum_{s=1}^{S} \nu_{s}$ and

$$
\begin{aligned}
(\forall s \in\{1, \ldots, S\}) \quad m_{s} & =\widehat{a}_{s} e^{-\widehat{k}_{s}} \frac{1-e^{-\widehat{k}_{s} T}}{1-e^{-\widehat{k}_{s}}} \\
v_{s} & =\sum_{t=1}^{T} v_{s, t}
\end{aligned}
$$

with, for every $t \in\{1, \ldots, T\}, v_{s, t}=\left(r_{s, t}-\widehat{a}_{s} e^{-\widehat{k}_{s} t}-\widehat{c}\right)^{2}$. Then, the estimate of $u$ is given by:

$$
(\forall s \in\{1, \ldots, S\}) \quad \widehat{u}_{s}=\frac{\widehat{a}_{s}}{\widehat{\alpha}} .
$$

Finally, the estimation process is completed by computing:

$$
\widehat{\sigma}^{2}=\frac{\sum_{(s, t) \in \mathbb{I}} \nu_{s}\left(v_{s, t}-\widehat{\alpha} \widehat{a}_{s} e^{-\widehat{k}_{s} t}\right)}{\sum_{(s, t) \in \mathbb{I}} \nu_{s}}
$$

where

$$
\mathbb{I}=\left\{(s, t) \in\{1, \ldots, S\} \times\{1, \ldots, T\} \mid v_{s, t}-\widehat{\alpha} \widehat{a}_{s} e^{-\widehat{k}_{s} t} \geq 0\right\} .
$$

\subsection{Refined estimation}

The main limitation of the previous moment based method is that the unknown parameters are not jointly estimated. Due to this fact, we may face large error propagation with respect to the estimation of some parameters. Thus, similarly to the work in [13], we propose to improve the moment based method results by resorting to an EM approach. The proposed noise identification procedure is summarized in Fig. 1.

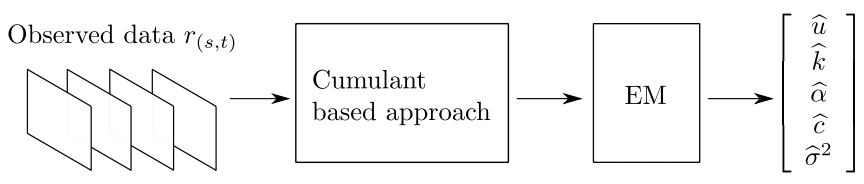

Fig. 1. Flowchart of the proposed noise identification method.

It must be emphasized that photobleaching was not discussed in [13], so that significant modifications of the EM algorithm were 
necessary to include this effect into this framework. More precisely, in the $(n+1)$-th maximization step, it can be shown that, for every $s \in\{1, \ldots, S\}$, the photobleaching rate $k_{s}^{(n+1)}=-\ln x^{(n+1)}$ where $x^{(n+1)}$ is the solution in $(0,1)$ of the polynomial equation:

$$
\begin{aligned}
& \left(1+T x^{T+1}-(T+1) x^{T}\right) \sum_{t=1}^{T} \mathrm{E}_{Q \mid R=r, \theta^{(n)}}\left[Q_{s, t}\right] \\
& =\left(1-x-x^{T}+x^{T+1}\right) \sum_{t=1}^{T} t \mathrm{E}_{Q \mid R=r, \theta^{(n)}}\left[Q_{s, t}\right] .
\end{aligned}
$$

We propose to compute $x^{(n+1)}$ from (15) using Halley's algorithm [22]. Then, $u$ can be derived as follows

$$
\begin{aligned}
(\forall s \in & \{1, \ldots, S\}) \quad u_{s}^{(n+1)}= \\
& \frac{1-x^{(n+1)}}{x^{(n+1)}\left(1-\left|x^{(n+1)}\right|^{T}\right)} \sum_{t=1}^{T} \mathrm{E}_{Q \mid R=r, \theta^{(n)}}\left[Q_{s, t}\right] .
\end{aligned}
$$

Finally, the maximization step is completed by update formulas for $\alpha^{(n+1)}, c^{(n+1)}$ and $\left(\sigma^{2}\right)^{(n+1)}$, which are unchanged with respect to the stationary case described in [13]. In the $n$-th expectation step, taking into account the photobleaching effect yields the following expressions:

$$
\begin{aligned}
& \mathrm{E}_{Q \mid R=r, \theta}\left[Q_{s, t}\right]=\frac{\zeta_{s, t}^{(n)}}{\eta_{s, t}^{(n)}} \\
& \zeta_{s, t}^{(n)}=\sum_{q_{s, t}=1}^{+\infty} e^{-\frac{\left(r_{s, t}-\alpha^{(n)} q_{s, t}-c^{(n)}\right)^{2}}{2\left(\sigma^{2}\right)^{(n)}}} \frac{\left(u_{s}^{(n)}\right)^{q_{s, t}}}{\left(q_{s, t}-1\right) !} e^{-t k_{s}^{(n)} q_{s, t}} \\
& \eta_{s, t}^{(n)}=\sum_{q_{s, t}=0}^{+\infty} e^{-\frac{\left(r_{s, t}-\alpha^{(n)} q_{s, t}-c^{(n)}\right)^{2}}{2\left(\sigma^{2}\right)^{(n)}}} \frac{\left(u_{s}^{(n)}\right)^{q_{s, t}}}{q_{s, t} !} e^{-t k_{s}^{(n)} q_{s, t}}
\end{aligned}
$$

\section{SIMULATION EXAMPLES}

In this section, we present experiments on both synthetic and real data in order to demonstrate the performance of the proposed approach. In particular, the simulations on synthetic data allow us to evaluate the reliability of our algorithm (Section 4.1) and the real data case study illustrates its potentials in microscopy applications (Section 4.2).

\subsection{Synthetic data validation}

We evaluate the proposed algorithms using $S=200$ randomly generated $u_{s}$ values uniformly distributed over $[0,150]$ and $k_{s}$ values uniformly distributed over $\left[10^{-4}, 10^{-3}\right]$. Signal $\left(r_{s, t}\right)_{1 \leq s \leq S, 1 \leq t \leq T}$ is generated according to (1) for $c=10, \alpha=30, \sigma^{2}=100$ and $T=180$. The algorithm performance is measured by the $\overline{\mathrm{SNR}}$ defined as the average of $10 \log _{10}(P)$ with $P=(S T)^{-1} \sum_{(t, s)}\left(a_{s} e^{-k_{s} t}\right)^{2} / \sum_{(t, s)}\left(a_{s} e^{-k_{s} t}-\widehat{a}_{s} e^{-\widehat{k}_{s} t}\right)^{2}$ computed over 50 noise realizations, where the estimated values are designated with a hat. Moreover, we examine the estimator properties by presenting the bias and standard deviation of the resulting noise parameters $\widehat{\alpha}, \widehat{c}$ and $\widehat{\sigma}$.

The results presented in Table 1 indicate that in the first step of the algorithm (Fig. 1) a majority of the unknown parameters are identified accurately. As expected, the estimates are further improved in the second step by EM, achieving high accuracy, indicated by high $\overline{\mathrm{SNR}}$ values, and low standard deviation of the estimated noise parameters.

\begin{tabular}{|c|c|c|c|c|c|c|c|}
\hline \multirow{2}{*}{ Method } & \multicolumn{2}{|c|}{$\hat{\sigma}$} & \multicolumn{2}{|c|}{$\hat{c}$} & \multicolumn{2}{|c|}{$\hat{\alpha}$} & \multirow{2}{*}{ SNR } \\
\cline { 2 - 7 } & bias & std & bias & std & bias & std & \\
\hline \hline Init. & 357.5 & 3.1 & 1.9 & 1.0 & -0.3 & 0.4 & 39.5 \\
\hline EM & 2.9 & 0.9 & 1.4 & 0.8 & -0.3 & 0.4 & 39.7 \\
\hline
\end{tabular}

Table 1. Synthetic data results.

\subsection{True data results}

In the real data case, noise parameter identification and true image intensity reconstruction follow registration of time lapse series of images. Images were acquired using a macro confocal laser scanning microscope (Leica TCS-LSI) from a cross-section through the rhizome of Convallaria majalis (Lily of the Valley). Measurements were done on images taken with the following settings: pinhole 1.06 airy, $800 \mathrm{~Hz}$ scan speed, PMT Offset $-4.3 \%$, PMT Gain 848, excitation line $488 \mathrm{~nm}$, and emission range $499 \mathrm{~nm}-690 \mathrm{~nm}$. The reported signal intensities at each location within the biological sample result from natural occurring autofluorescence caused by different compounds like lignin and other phenolics. As such, the process of bleaching and decrease in image intensity is complex and cannot be fitted with a mono-exponential decay [23].

The processed time lapse sequence consists of 180 images with 12-bit resolution of size $128 \times 128$, which translates into $T=180$ and $S=16384$. The first and last images of the sequence are illustrated in Fig. 2 (a) and Fig. 2 (b), respectively. One can observe that, due to the photobleaching effect, some parts of the image $r_{s, 1}$ (Fig. 2 (a)) are not visible in the last image $r_{s, 180}$ (Fig. 2 (b)), e.g. quadrant 4 . Weakly fluorescing components are lost when calculating the mean over all realizations (Fig. 2 (c)), while they are well preserved and less oversmoothed when applying our algorithm (Fig. 2 (d)). Besides these visual results, our algorithm also allows us to establish the parameters of the noise model (1), which are given as $25.8 \mathcal{P}\left(\widehat{u}_{s} e^{-\widehat{k}_{s} t}\right)+\mathcal{N}(8,119)$. The plots in Fig. 3 illustrate the change of the measured and reconstructed signals along $t$, while $s$ is fixed. They show the variety of acquired signals. For instance, the signal presented in Fig. 3(b) corresponds to a weak photobleaching effect, while in Fig. 3(c), a strong photobleaching effect is identified. One can observe that the bleaching curves are a good fit for the series of measured data points. The identified bleaching rates $\widehat{k}_{s}$ belong to the set $\left(0,3.9 \times 10^{-6}\right] \mathrm{Hz}$, and $\widehat{u}_{s}$ values lie in $[0,147]$. This example illustrates the properties of our method applied to a fluorescence imaging system.

\section{CONCLUSIONS}

The present study has addressed the problem of Poisson-Gaussian noise parameter identification from time-lapse data, taking into account the photobleaching effect. We have proposed new, fully automated, two-step approach dealing with this problem. Our experiments illustrate that this approach leads to a very accurate image reconstruction as illustrated by Fig. 3(d). The proposed algorithm has a direct application in the calibration of fluorescence imaging systems like confocal macroscopy and as a preliminary step in image restoration procedures. One should note that one limitation of our proposed approach is the requirement for a stationary specimen. To overcome this problem, a motion compensation scheme can be used. 


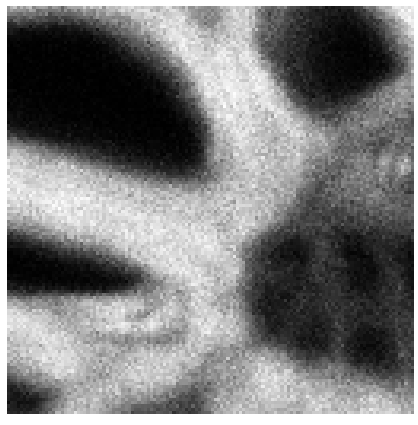

(a) $r_{s, 1}$

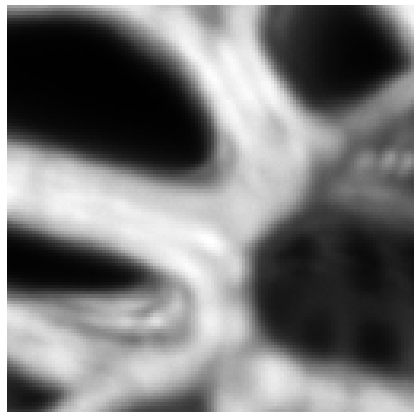

(c) $\frac{1}{T} \sum_{t=1}^{T} r_{s, t}$

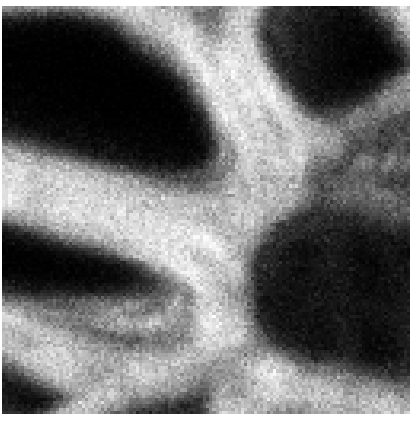

(b) $r_{s, 180}$

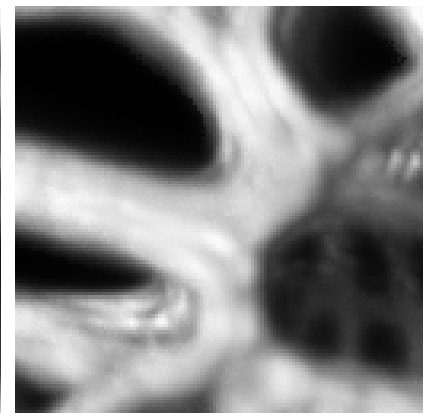

(d) reconstructed $\widehat{u}_{s}$
Fig. 2. (a,b) illustrate first and last observations of time series images $r_{s, t}$, (c) mean over all realizations and (d) reconstructed image.

\section{REFERENCES}

[1] F. Luisier, T. Blu, and M. Unser, "Image denoising in mixed Poisson-Gaussian noise," IEEE Transactions on Image Processing, vol. 20, no. 3, pp. 696-708, Mar. 2011.

[2] F. Benvenuto, A. La Camera, C. Theys, A. Ferrari, H. Lantéri, and M. Bertero, "The study of an iterative method for the reconstruction of images corrupted by Poisson and Gaussian noise," Inverse Problems, vol. 24, no. 3, 2008, 20 pp.

[3] J. M. Zwier, G. J. Van Rooij, J. W. Hofstraat, and G. J. Brakenhoff, "Image calibration in fluorescence microscopy," Journal of Microscopy, vol. 216, no. 1, pp. 15-24, 2004.

[4] J. B. Pawley, "Sources of noise in three-dimensional microscopical data sets," in Three-Dimensional Confocal Microscopy: Volume Investigation of Biological Specimens, J. Stevens, L. Mills, and J. Trogadis, Eds., pp. 47-94. Academic Press, San Diego, CA., 1994.

[5] D. Sage, F. R. Neumann, F. Hediger, S. M. Gasser, and M. Unser, "Automatic tracking of individual fluorescence particles: application to the study of chromosome dynamics," IEEE Trans. Image Process., vol. 14, pp. 1372-1383, 2005.

[6] I. Rodrigues and J. Sanches, "Fluorescence microscopy imaging denoising with log-euclidean priors and photobleaching compensation," in Proc. Int. Conf. Image Process., Cairo, Egypt, 2009, pp. 809-812.

[7] B. Zhang, Contributions à la microscopie à fluorescence en imagerie biologique : modélisation de la PSF, restauration d'images et détection super-résolutive, Phd thesis, TELECOM Paris, Nov. 2007.

[8] T. Bernas, D. Barnes, E. K. Asem, J. P. Robinson, and B. Rajwa, "Precision of light intensity measurement in biological optical microscopy," Journal of Microscopy, vol. 226, no. 2, pp. 163-174, 2007.

[9] S. Delpretti, F. Luisier, S. Ramani, T. Blu, and M. Unser, "Multiframe sure-let denoising of timelapse fluorescence microscopy images," in 5th IEEE International Symposium on Biomedical Imaging: From Nano to Macro (ISBI), Paris, France, 2008, pp. 149-152.

[10] J. Boulanger, J. B. Sibarita, C. Kervrann, and P. Bouthemy, "Non-parametric regression for patch-based fluorescence microscopy image sequence denoising," in 5th IEEE International Symposium on Biomedical Imaging: From Nano to Macro (ISBI), Paris, France, 2008, pp. 748-751.

[11] P. Paul, H. Duessmann, T. Bernas, H. Huber, and D. Kalamatianos, "Automatic noise quantification for confocal fluorescence microscopy images," Computerized Medical Imaging and Graphics, vol. 34, no. 6, pp. 426-434, Sep. 2010.

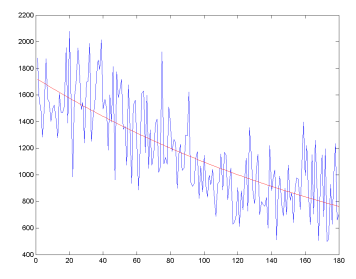

(a) $25.8 \times 67 e^{-1.6 \times 10^{-6} t}+8$

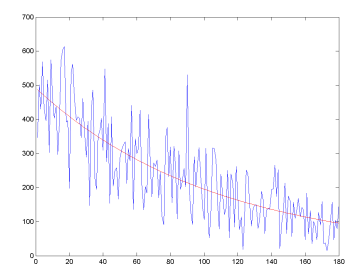

(c) $25.8 \times 19 e^{-3.6 \times 10^{-6} t}+8$

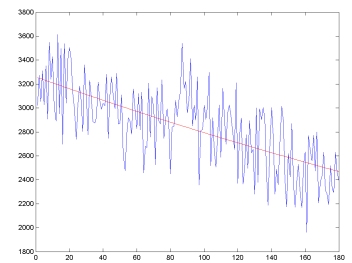

(b) $25.8 \times 126 e^{-5.7 \times 10^{-7} t}+8$

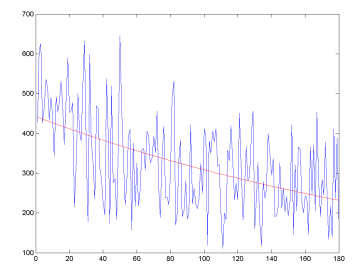

(d) $25.8 \times 17 e^{-1.4 \times 10^{-6} t}+8$
Fig. 3. (a,b,c,d) illustrate time variations for fixed $s$. The true data are plotted in blue and the reconstructed one (using formula $\left.\widehat{\alpha} \widehat{u}_{s} e^{-\widehat{k}_{s} t}+\widehat{c}\right)$ in red.

[12] K. M. Kedziora, J. H.M. Prehn, J. Dobrucki, and T. Bernas, "Method of calibration of a fluorescence microscope for quantitative studies," Journal of Microscopy, vol. 244, no. 1, pp. 101-111, 2011.

[13] A. Jezierska, C. Chaux, J.-C. Pesquet, and H. Talbot, "An EM approach for Poisson-Gaussian noise modeling," in European Signal Processing Conference (EUSIPCO), Barcelona, Spain, Aug. 2011, pp. 2244-2248.

[14] P. Pankajakshan, A. Dieterlen, G. Engler, Z. Kam, L. Blanc-Féraud, J. Zerubia, and J.C. Olivo-Marin, "Wavefront sensing for aberration modeling in fluorescence MACROscopy," in Proc. IEEE International Symposium on Biomedical Imaging (ISBI), Chicago, USA, Apr. 2011, pp. 905 - 908.

[15] N. Vicente, J. Zamboni, J. Adur, E. Paravani, and V. Casco, "Photobleaching correction in fluorescence microscopy images," Journal of Physics: Conference Series, vol. 90, no. 1, 2007, 8 pp.

[16] A. J. Berglund, "Nonexponential statistics of fluorescence photobleaching," $J$. Chem. Phys., vol. 121, no. 7, pp. 2899-2903, 2004.

[17] L. Song, E. Hennink, I. Young, and H. Tanke, "Photobleaching kinetics of fluorescein in quantitative fluorescence microscopy," Biophysical Journal, vol. 68, no. 6, pp. 2588-2600, Jun. 1995.

[18] C. Biernacki, G. Celeux, and G. Govaert, "Choosing starting values for the EM algorithm for getting the highest likelihood in multivariate Gaussian mixture models.," Computational Statistics \& Data Analysis, vol. 41, no. 3-4, pp. 561-575, 2003.

[19] J. Pereira, R. Celso, L. Marques, and J. Justino da Costa, "An empirical comparison of EM initialization methods and model choice criteria for mixtures of skew-normal distributions," in 19 SINAPE - Simpósio Nacional de Probabilidade e Estatística, 2010.

[20] S. Huda, R. Ghosh, and J. Yearwood, "A variable initialization approach to the EM algorithm for better estimation of the parameters of hidden Markov model based acoustic modeling of speech signals," in Advances in Data Mining. Applications in Medicine, Web Mining, Marketing, Image and Signal Mining, Petra Perner, Ed., vol. 4065 of Lecture Notes in Computer Science, pp. 416-430. Springer Berlin / Heidelberg, 2006, 10.1007/11790853_33.

[21] J. C. Lagarias, J. A. Reeds, M. H. Wright, and P. E. Wright, "Convergence properties of the Nelder-Mead Simplex method in low dimensions," SIAM Journal of Optimization, vol. 9, pp. 112-147, 1998

[22] W. Gander, "On Halley's iteration method," The American Mathematical Monthly, vol. 92, no. 2, pp. 131-134, 1985.

[23] M. Talhavini and T.D.Z. Atvars, "Photostability of xanthene molecules trapped in poly(vinyl alcohol) (pva) matrices," Journal of Photochemistry and Photobiology A: Chemistry, vol. 120, no. 2, pp. $141-149,1999$. 